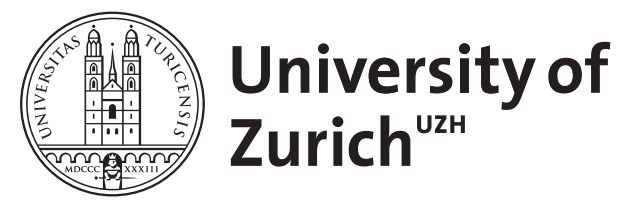

\title{
Gout in pediatric renal transplant recipients
}

Trück, Johannes ; Laube, G F ; von Vigier, R O ; Goetschel, P

\begin{abstract}
Clinical gout has rarely been described after pediatric renal transplantation (RTx), although asymptomatic hyperuricemia is common in these patients. We describe three male pediatric patients who presented with gouty arthritis 7-8.5 years following RTx. Since receiving allopurinol, all patients had been free of gouty symptoms. To prevent severe bone marrow depletion, the dosage of azathioprine, an immunosupressant drug, was reduced by $50 \%$ to prevent interaction with allopurinol. Because atypical presentation of gout can occur, a high index of suspicion is needed to allow appropriate diagnosis of this disease in patients with skeletal pain after RTx.
\end{abstract}

DOI: https://doi.org/10.1007/s00467-010-1599-6

Posted at the Zurich Open Repository and Archive, University of Zurich ZORA URL: https://doi.org/10.5167/uzh-44525

Journal Article

Published Version

Originally published at:

Trück, Johannes; Laube, G F; von Vigier, R O; Goetschel, P (2010). Gout in pediatric renal transplant recipients. Pediatric Nephrology, 25(12):2535-2538.

DOI: https://doi.org/10.1007/s00467-010-1599-6 


\title{
Gout in pediatric renal transplant recipients
}

\author{
Johannes Trück • Guido F. Laube • \\ Rodo O. von Vigier • Philippe Goetschel
}

Received: 30 March 2010 /Revised: 7 June 2010 / Accepted: 16 June 2010 / Published online: 18 July 2010

(C) IPNA 2010

\begin{abstract}
Clinical gout has rarely been described after pediatric renal transplantation (RTx), although asymptomatic hyperuricemia is common in these patients. We describe three male pediatric patients who presented with gouty arthritis 7-8.5 years following RTx. Since receiving allopurinol, all patients had been free of gouty symptoms. To prevent severe bone marrow depletion, the dosage of azathioprine, an immunosupressant drug, was reduced by $50 \%$ to prevent interaction with allopurinol. Because atypical presentation of gout can occur, a high index of suspicion is needed to allow appropriate diagnosis of this disease in patients with skeletal pain after RTx.
\end{abstract}

Keywords Kidney transplantation - Gout · Hyperuricemia . Children · Azathioprine $\cdot$ Allopurinol

\section{Introduction}

Hyperuricemia and gout are common problems among adult renal transplant recipients $[1,2]$. Gout is a painful disorder caused by an inflammatory reaction to the presence of monosodium urate crystals in the joint fluid and periarticular

J. Trück $\cdot$ P. Goetschel $(\square)$

Department of Pediatrics, Triemli Hospital Zurich,

Birmensdorferstrasse 497,

8063 Zurich, Switzerland

e-mail: philippe.goetschel@triemli.stzh.ch

G. F. Laube

Nephrology Unit, University Children's Hospital Zurich,

Zurich, Switzerland

R. O. von Vigier

Pediatric Nephrology,

University Children's Hospital Bern Inselspital,

Bern, Switzerland tissue, which in turn is the result of longstanding hyperuricemia [3]. In pediatric renal transplant recipients, asymptomatic hyperuricemia occurs most frequently in those patients who have survived a long time after transplantation, and it is probably a consequence of chronic renal transplant dysfunction [4]. Gout, however, is rare in these patients. We report on three patients who presented with partly atypical manifestation of gouty arthritis several years after undergoing pediatric renal transplantation (RTx).

\section{Patients}

Three male patients were found to have gouty arthritis 7 8.5 years following RTx. All patients had impaired renal function due to chronic transplant nephropathy.

Patient A, a 15-year-old boy with sporadic steroidresistant focal segmental glomerulosclerosis, suffered from clinical gout, with the first episode occurring 8 years after RTx. The clinical manifestation was typical arthritis of the metatarsophalangeal joint of the left big toe [4].

Patient B, a 17-year-old male adolescent with renal hypodysplasia, presented with fever, chills, and painful swelling localized above the head of the second metatarsal bone 8.5 years after RTx. Following plain radiography and magnetic resonance imaging (Fig. 1) of the right forefoot, he underwent biopsy to exclude osteomyelitis or septic arthritis. Blood culture as well as culture and eubacterial PCR of a bone specimen yielded negative results. Histopathological examination ultimately revealed gouty synovitis (Fig. 2).

Patient C, a 12-year-old boy with congenital nephrotic syndrome of the Finnish type, showed a typical clinical presentation with gouty arthritis of the metatarsophalangeal joint of the left big toe 7 years after his second renal transplantation (the first transplant was rejected 9 days after 
Fig. 1 a Plain radiograph of the right forefoot (Patient B): area of osteolysis (arrow) in the head of the second metatarsal bone. $\mathbf{b}$ Magnetic resonance imaging of the right forefoot (Patient B): edema of the soft tissue and of the bone marrow (arrow) in the diaphysis and the head of the second metatarsal bone with sclerotic destruction on the lateral border; effusion in the second metatarsophalangeal joint with synovial enhancement
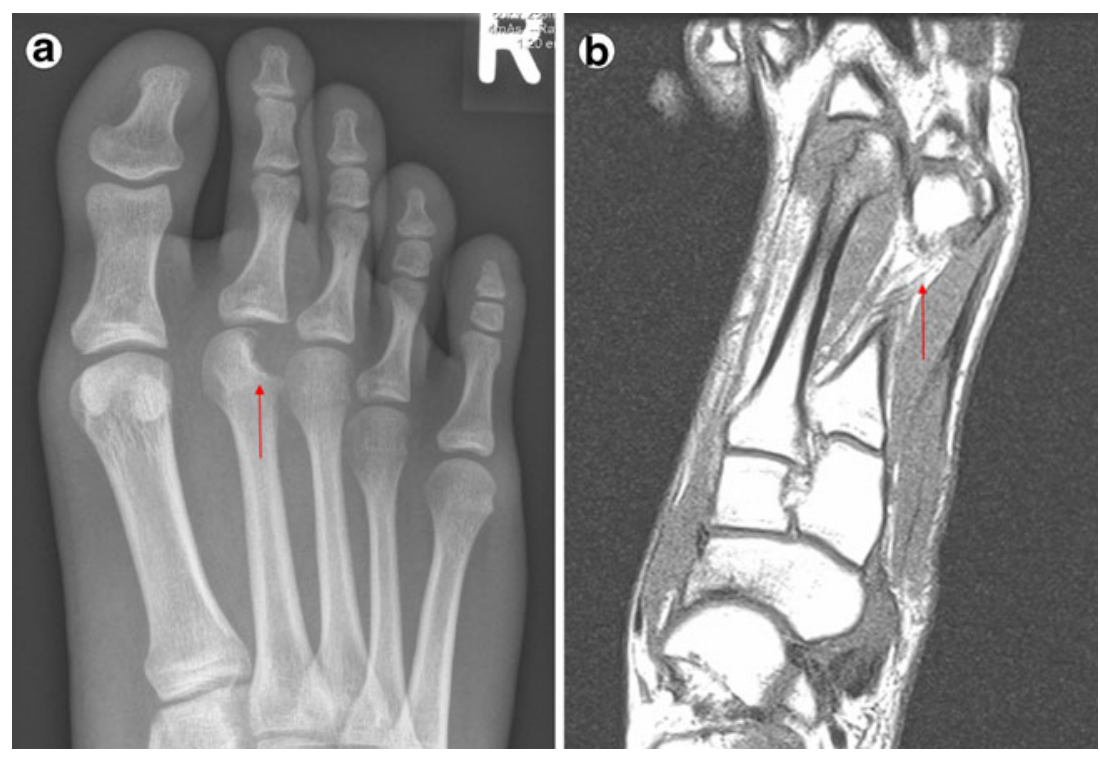

a living donor kidney transplantation at 2 years of age). The most important clinical and laboratory findings as well as the drug therapy are listed in Table 1 . After receiving longterm allopurinol (2-6 mg/kg/day), all patients were free of gouty symptoms.

\section{Discussion}

Hyperuricemia and gout are frequent complications in adult renal transplant recipients, with reported prevalences of up to 80 and $10 \%$, respectively $[1,2]$. Immunosuppressive agents, such as ciclosporin and tacrolimus, play a key role in inducing hyperuricemia and gout [3]. Hyperuricemia also seems to be common in pediatric patients. In one study, elevated uric acid concentrations were found in $47 \%$ of the pediatric patients after a median period of almost 5 years post-transplantation [4]. Clinical gout, however, is rare in these children [5]. We report here the history of three patients with new-onset clinical gout. Our aim was to alert physicians to the possibility of this rare clinical entity presenting in pediatric patients after RTx.

In general, gout has a predilection for the first metatarsophalangeal joint as well as wrists, knees, and elbows [2]. Proximal distribution of gouty arthritis involving the hips, shoulders, and sacroiliac joints as well as gouty enthesitis have also been described [6]. Onset is abrupt, and the affected joints are erythematous, warm, swollen, and tender. Patients A and C presented with classical podagra, characterized as an acute gouty arthritis of the first metatarsophalangeal joint. Hyperuricemia was present in both and supported the clinical diagnosis of gout. However, in patients with more pronounced inflammatory manifestations, gouty arthritis should be distinguished from septic arthritis, and immunosuppressive therapy does not seem to mitigate symptoms in all transplant patients [2]. This latter observation is upheld in patient $\mathrm{B}$, who presented with fever, chills and less typical localization of joint involve-

Fig. 2 Microscopic aspects of gouty tophus (Patient B). a Clusters of eosinophilic urate crystals (arrow) surrounded by histiocytes and macrophages [hematoxylin/eosin stain (HE), $10 \times]$. b Higher magnification presenting detritus and rare urate crystals (arrow) inducing an inflammation of the foreign type (HE, 40×)
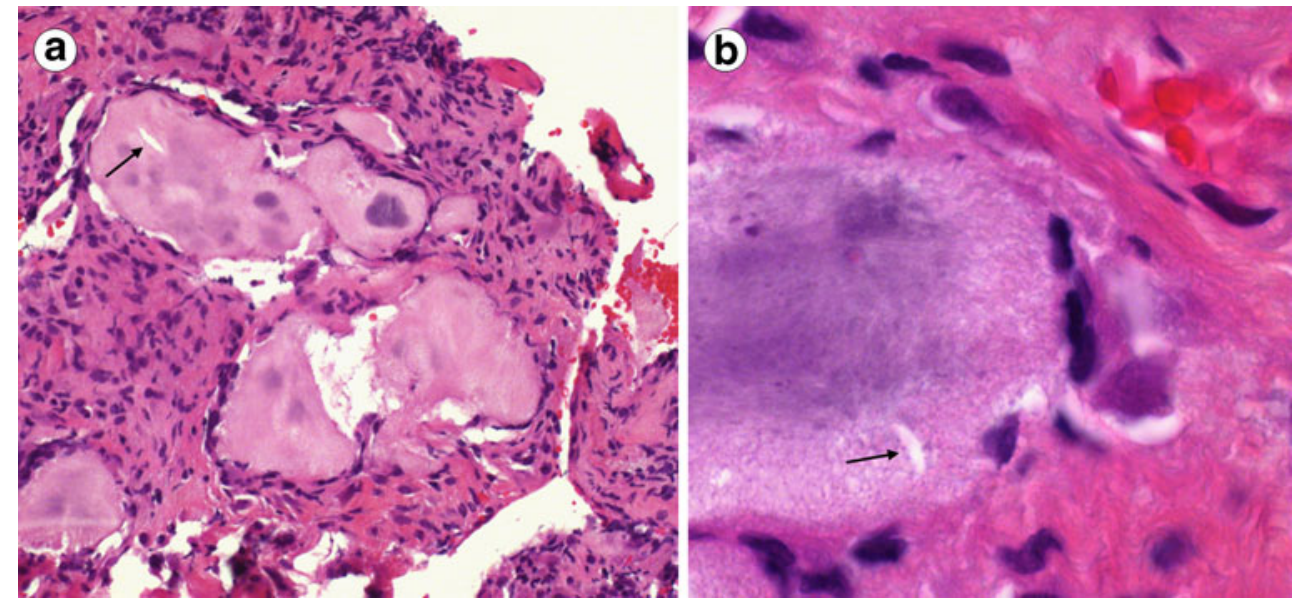
Table 1 Clinical, pharmacological and laboratory findings of the three patients

\begin{tabular}{llll}
\hline Patient data & Patient A & Patient B & Patient C \\
\hline Diagnosis & FSGS & RH & CNS Finnish type \\
Age (years) & 15 & 17 & 12 \\
Time post RTx (years) & 8 & 8.5 & 7 \\
Affected joint & MTP I & MTP II & MTP I \\
Plasma creatinine $\left(\mu \mathrm{mol}^{\mathrm{l}}\right)$ & 217 & 175 & 218 \\
eGFR $\left(\mathrm{ml} / \mathrm{min} / 1.73 \mathrm{~m}^{2}\right)^{\mathrm{a}}$ & 29 & 39 & 29 \\
Uric acid $(\mu \mathrm{mol} / \mathrm{l})$ & 929 & 709 & 611 \\
Immunosuppression & CSA, AZA, PDN & CSA, AZA & SIR, MMF, PDN \\
Diuretics & Furosemide & - & - \\
\hline
\end{tabular}

FSGS, Steroid-resistant focal segmental glomerulosclerosis; RH, renal hypodysplasia; CNS, Congenital nephrotic syndrome; RTx, renal transplantation; eGFR, estimated glomerular filtration rate; MTP, metatarsophalangeal joint; CSA, ciclosporin A, SIR, sirolimus; AZA, azathioprine; MMF, mycophenolate mofetil; PDN, prednisolone

${ }^{a}$ Determined by the Haycock-Schwartz formula

ment. Therefore, this patient was further investigated to search for infectious complications. Notably, gout and septic arthritis can coexist within the same joint [7]. Pseudomonas aeruginosa was not present in patient B; infection by this microorganism has been described in patients as a complication following (unnoticed) puncture wounds while wearing sneakers [8]. In this patient, gout was finally diagnosed by histopathological examination of biopsy specimens.

In the vast majority of patients with gouty arthritis, hyperuricemia is a prerequisite, at least at some point in their disease [3], although it is worth noting that some patients do have normal uric acid concentrations during an acute attack of gout [9]. Several factors may contribute to the occurrence of hyperuricemia in renal transplant recipients: (1) ciclosporin; (2) diuretics; (3) hypertensive nephropathy [2], and (4) chronic renal transplant dysfunction [4]. In pediatric renal transplant recipients, however, the hyperuricemic effect of ciclosporin appears to be less relevant than in adults. In the former, plasma uric acid concentration significantly and positively correlates with the length of the time span after renal transplantation and inversely correlates with renal function [4]. The impact of impaired renal function on the development of hyperuricemia long after RTx and the subsequent existence of gout was apparent in our three patients. Additionally, only one of the present patients received diuretic treatment (Patient A).

The treatment of gout in transplant recipients is challenging due to the potential for drug interactions and the risk of side effects. The standard treatment of acute attacks of gout consists of rest, application of ice to the affected joint, and a prescription for colchicine, nonsteroidal anti-inflammatory drugs (if not precluded by limited renal function), or both. Alternatively, and probably more suitable in renal transplant recipients, a short-term course of systemic corticosteroids (e.g. prednisolone $1 \mathrm{mg} /$ $\mathrm{kg} /$ day for 4-7 days) or intra-articular corticosteroid injections are of great value in terms of improving the symptoms [3]. Xanthine oxidase inhibitors, such as allopurinol, are also a very effective treatment option, especially as a prophylaxis of gout. Since the metabolism of azathioprine is partly dependent on xanthine oxidase, coadministration of these drugs results in an increased risk of severe bone marrow toxicity. Therefore, some treatment protocols avoid this combination [10]. If, however, azathioprine and allopurinol are co-administered, the dose of the former has to be reduced by approximately $50 \%$, and careful monitoring of blood cell counts is mandatory. Alternatively, a switch from azathioprine to mycophenolate mofetil can be considered, as this will avoid potential toxic drug interactions. Further therapeutic options in these patients are colchicine and uricosuric agents [11]. In our patients, treatment with allopurinol and co-administration of a reduced dose of azathioprine in two of them were tolerated without signs of toxicity.

In conclusion, despite the common occurrence of hyperuricemia in pediatric renal transplant recipients, gout remains rare in these patients. Clinical gout usually occurs several years after RTx, and presentation might be atypical. Consequently, diagnosis can be challenging. Because of possible drug interactions and the risk of side effects, treatment should be individually managed by a pediatric nephrologist.

Acknowledgments We thank Dr. Philip Went for providing photos of Fig. 2 and Dr. Jan Janzen for helping with the interpretation of the histopathology.

Conflicts of interest The authors declare that they have no conflicts of interest in writing the article. 


\section{References}

1. Abbott KC, Kimmel PL, Dharnidharka V, Oglesby RJ, Agodoa LY, Caillard S (2005) New-onset gout after kidney transplantation: incidence, risk factors and implications. Transplantation 80:13831391

2. Clive DM (2000) Renal transplant-associated hyperuricemia and gout. J Am Soc Nephrol 11:974-979

3. Richette P, Bardin T (2010) Gout. Lancet 375:318-328

4. Sparta G, Kemper MJ, Neuhaus TJ (2006) Hyperuricemia and gout following pediatric renal transplantation. Pediatr Nephrol 21:1884-1888

5. Pela I, Seracini D, Lavoratti G, Materassi M (1999) Acute gouty arthritis in adolescents with renal transplants. Pediatr Med Chir $21: 135-137$
6. Cohen MR (1994) Proximal gout following renal transplantation. Arthritis Rheum 37:1709

7. Yu KH, Luo SF, Liou LB, Wu YJ, Tsai WP, Chen JY, Ho HH (2003) Concomitant septic and gouty arthritis-an analysis of 30 cases. Rheumatology (Oxford) 42:1062-1066

8. Fisher MC, Goldsmith JF, Gilligan PH (1985) Sneakers as a source of Pseudomonas aeruginosa in children with osteomyelitis following puncture wounds. J Pediatr 106:607-609

9. McCarty DJ (1994) Gout without hyperuricemia. JAMA 271:302-303

10. Perez-Ruiz F, Gomez-Ullate P, Amenabar JJ, Zarraga S, Calabozo M, Herrero-Beites AM, Nolla JM (2003) Long-term efficacy of hyperuricaemia treatment in renal transplant patients. Nephrol Dial Transplant 18:603-606

11. Stamp L, Searle M, O'Donnell J, Chapman P (2005) Gout in solid organ transplantation: a challenging clinical problem. Drugs 65:2593-2611 